\title{
ORIGINAL
}

\section{VALORACIÓN DEL ÍNDICE DE SALUD SF-36 APLICADO A USUARIOS DE PRO- GRAMAS DE METADONA. VALORES DE REFERENCIA PARA LA COMUNIDAD AUTÓNOMA VASCA}

Ioseba Iraurgi Castillo (1-2), Mónica Póo (3) e Iñaki Márkez Alonso (3)

(1) Módulo de Asistencia Psicosocial de Rekalde.

(2) Asociación Vasca para el Estudio e Investigación de las Adicciones

(3) Eximen. Investigación en Salud Mental y Drogodependencias

\section{RESUMEN}

Fundamento: Los cuestionarios que miden la salud percibida sirven en la práctica clínica para determinar el impacto de la enfermedad y los bre bilidad se ha propuesto obtener normas de referencia. El objetivo principal del estudio ha sido obtener valores de referencia del cuestionario de salud SF-36 aplicado a usuarios de Programas de Mantenimiento con Metadona (PMM) en la Comunidad Autónoma Vasca (CAV).

Métodos: Diseño transversal realizado en el año 2000 sobre una muestra estratificada aleatoria de usuarios PMM de la CAV ( $\mathrm{n}=726$ ). Se utilizó el cuestionario de salud SF-36, y se calcularon datos de tendencia central, dispersión y percentiles para generar los valores de referencia en función del tiempo de permanencia en el programa de metadona.

Resultados: Los valores alfa de Cronbach se situaron por encima de 0,7 , siendo el porcentaje de no respuestas inferior al $1 \%$. En todas las dimensiones del SF-36 las puntuaciones fueron inferiore para los usuarios de PMM respecto a los valores poblacionales de referencia $(\mathrm{p}<0,001)$. El estado de salud de los participantes era peor entre los que iniciaban el tratamiento que entre los que llevaban más de seis meses en el PMM (diferencias significativas en todas las dimensiones, $\mathrm{p}<0,001$ )

Conclusiones: El SF-36 como medida del estado de salud muestra una alta fiabilidad y es capaz de discriminar pacientes en estadios diferentes de tratamiento. Los valores obtenidos podrían tomarse como valores de referencia para ayudar a monitorizar la progresión de mejora o la respuesta al tratamiento en personas incluidas en programas de metadona.

Palabras clave: Salud percibida. Cuestionario. SF-36. Valores de referencia. Metadona. Drogadicción. Heroína.

Correspondencia:

Ioseba Iraurgi Castillo

Módulo de Asistencia Psicosocial de Rekalde

Camilo Villabaso 24 lonja / 48002 Bilbao

Correo electrónico: iraurgi@euskalnet.ne

\section{ABSTRACT}

Evaluation of the SF-36 Health Index Applied to Methadone Maintenance Program Users. Reference Values for the Basque Autonomous Community, Spain

Background: The questionnaires that measure perceived health are used in clinical practice to determine the impact of illnesses and the benefits of treatments. Population-based norms have been proposed to increase their interpretability. The aim of this paper was to obtain reference values for users of Methadone Maintenance Programs (MMP) in the Basque Autonomous Community (BAC)

Methods: Cross-sectional study carried out in 2000 on a stratified random sample of users included in MMP of BAC $(n=726)$. SF36 Health Survey was used; central trend, dispersion and percentile data were estimated for each of the SF- 36 dimension scores to generate reference values according the dwell time in the methadone program.

Results: All Cronbach's alpha coefficients were higher than 0.7 , and the percentage or non-responds were lower $1 \%$. The MMP user had lower punctuations in all dimensions of SF-36 that population values of reference $(\mathrm{p}<0.001)$. The health status of the participants was worse among those than they began the treatment that among those that took more than six months in the MMP; the differences in all SF-36 dimensions scores were statistically significant $(\mathrm{p}<0.001)$.

Conclusions: The SF-36 as a measure of health status in MMP population shows a high internal consistence, and is able to discriminate patients with different stages of treatment evolution. Results presented should be considered the population-based norms for monitoring the improvement progression or response to treatment in patients included in MMP.

Key words: Self-perceived health. Questionnaire. Reference values. Methadone. Drug addiction. Heroine. 


\section{INTRODUCCIÓN}

El tratamiento por sustitución o mantenimiento con metadona es en la actualidad la forma más difundida y efectiva de tratamiento para la adicción a opiáceos ${ }^{1,4}$. $\mathrm{La}$ efectividad clínica de los Programas de Mantenimiento con Metadona (PMM) es tradicionalmente medida a través de los criterios llamados duros, tales como la consecución de la abstinencia de la droga que genera la demanda y la disminución del consumo de otras sustancias, el nivel de retención en el programa, la reducción de la criminalidad, el aumento de la actividad laboral o la reducción de la morbi-mortalidad ${ }^{5,6}$. No obstante, durante la última década los evaluadores e investigadores han puesto un especial énfasis en medidas de resultado clínico basadas en la apreciación subjetiva y declaración de los propios usuarios de los servicios de salud ${ }^{6,8}$. Estas medidas, basadas fundamentalmente en cuestionarios con características psicómetricas bien fundamentadas, tratan de explorar aspectos tales como el estado de salud, la calidad de vida percibida o la satisfacción con los servicios y la atención recibida ${ }^{9,14}$.

Entre los instrumentos de medida de la salud percibida más ampliamente utilizados en la evaluación de los PMM $^{15}$ se halla el Cuestionario de Salud SF-36 ${ }^{16,20}$. Éste es un cuestionario genérico de salud diseñado tanto para su uso individual como colectivo. $\mathrm{Su}$ calidad ha hecho que sea uno de los índices de salud más utilizados por la comunidad científica, lo que permite la comparación nacional e internacional de los resulta$\operatorname{dos}^{21,24}$.

La utilización de este tipo de cuestionarios estandarizados, aplicados a muestras representativas de una determinada población, permite la generación de valores o normas poblacionales que se emplean para estimar la salud percibida de la población y su evolución en el tiempo, y sirven asimismo de referencia para la comparación de las diferencias en el estado de salud (calidad de vida) según las patologías o tratamientos y/o monitorizar procesos de tratamiento ${ }^{13}$. Por ello, las normas poblacionales de la salud percibida podrían usarse para evaluar cualitativa y cuantitativamente el impacto de la enfermedad y el beneficio de los procesos sanitarios $^{11,13,22,25}$.

El presente trabajo trata de abordar distintos objetivos. El primero afronta la valoración de las características psicométricas del SF-36 cuando es aplicado a una muestra de usuarios de PMM. Como segundo objetivo se comparan los valores obtenidos en dicha muestra con los correspondientes a los valores normativos dados para la adaptación a la población española ${ }^{21,22}$ y los valores de referencia para la población de la Comunidad Autónoma Vasca $(\mathrm{CAV})^{23}$. Finalmente, y como objetivo principal, se busca la obtención de los valores de referencia del Cuestionario de Salud SF-36 para población usuaria de Programas de Mantenimiento con Metadona en la Comunidad Autónoma Vasca en función del tiempo de permanencia en el programa de tratamiento.

\section{SUJETOS Y MÉTODOS}

La población objeto de estudio estaba constituida por las personas con diagnóstico de dependencia de opioides incluidas durante el año 2000 en los PMM de la Comunidad Autónoma Vasca. Para la extracción de una muestra representativa de esta población se tomó como referencia el volumen de personas asistidas en este programa en diciembre de 1998, que era de 1.946 usuarios según el registro de casos psiquiátricos de la Dirección de Salud Mental de Osakideza (Servicio Vasco de Salud ${ }^{26}$. A partir de este censo de población de usuarios de PMM se consideró necesario un tamaño muestral de 504 usuarios/as para el supuesto más desfavorable $(\mathrm{p}=\mathrm{q}=50)$ y un error de estimación máximo de $\pm 4 \%$. Asimismo, se planificó un diseño muestral estratificado en función de la 
distribución de usuarios de PMM por provincia y tipo de centro de dispensación (Centro de Tratamiento de Toxicomanías -CTTo en farmacia).

Se contactó con los centros dispensadores y en función de las cuotas de estratificación se realizó una selección aleatoria de los participantes. El trabajo de campo se realizó en dos fases; la primera entre enero y marzo del año 2000, y la segunda entre septiembre y octubre del mismo año. Se entrevistó a un total de 726 usuarios, recogiéndose un nivel de rechazo del 5,3\% (41 sujetos se negaron a participar). El reparto de casos en función del tipo de servicio (CTT vs Farmacias) fue del $54,5 \%$ y del $45,6 \%$ respectivamente, y las cuotas de participación por provincias fueron bastante aproximadas a las correspondientes a la población.

Todos los sujetos fueron entrevistados mediante cuestionario estructurado por personal entrenado a tal efecto o por personal sanitario, también entrenado. Fue criterio de inclusión el seguimiento clínico en algún centro sanitario acreditado y la voluntariedad del paciente para realizar las entrevistas, que fueron efectuadas previo consentimiento informado. El cuestionario utilizado incluía preguntas destinadas a conocer hábitos de salud y prevención de riesgos, consumo de sustancias durante la permanencia en el PMM, cambios en distintas áreas psicosociales (relaciones familiares, empleo, delincuencia, etc.), ... También se incluía la versión española del cuestionario de Salud SF36 con el objetivo de valorar el estado de salud percibido por los propios participantes.

\section{Cuestionario de Salud SF-36}

El cuestionario de salud SF-36 $6^{16,19}$ fue desarrollado para su uso en el Estudio de Resultados Médicos (Medical Outcome Study-MOS) a partir de una amplia batería de cuestionarios que incluían 40 conceptos relacionados con la salud. A partir de este estudio han surgido diferentes versiones del SF, de 12, 20, 36, 42, ... ítems, pero la más ampliamente utilizada en investigación y evaluación de los resultados clínicos ha sido el formato de 36 ítems. Es aplicable tanto en población general como en colectivos clínicos, en estudios ya sean descriptivos o de evaluación ${ }^{27}$. La adaptación del cuestionario para su uso en España ha sido realizada por el equipo de Alonso $^{21,28}$, mostrando niveles adecuados de validez, fiabilidad y equivalencia cultural con otras versiones de otros países $^{24}$. Asimismo, existen valores de referencia de las puntuaciones del SF-36 tanto en población general española ${ }^{22}$ como en personas residentes en la Comunidad Autónoma Vasca ${ }^{23}$.

Los ítems del cuestionario informan de estados tanto positivos como negativos de la salud física y del bienestar emocional, identificando ocho dimensiones de salud: función física (FF), limitaciones de rol por problemas físicos (RF), dolor corporal (D), salud general (SG), vitalidad (V), función social (FS), limitaciones de rol por problemas emocionales (RE) y salud mental (SM). Además de estas ocho escalas de salud, se incluye un ítem que evalúa el concepto general de cambios en la percepción del estado de salud actual respecto a como era un año antes (CS). En el Anexo se describen las dimensiones de salud evaluadas por el SF-36 y sus respectivas interpretaciones de acuerdo con los resultados bajos o altos por cada escala.

Para cada dimensión del SF-36, los ítems se codifican, agregan y transforman en una escala con recorrido desde 0 (peor estado de salud) hasta 100 (mejor estado de salud) utilizando los algoritmos e indicaciones que ofrece el manual de puntuación e interpretación del cuestionario ${ }^{16,28}$. Por tanto, una mayor puntuación en las diferentes dimensiones indica un mejor estado de salud y/o una mejor calidad de vida. 
Anexo 1

Subescalas del índice de salud SF-36 e interpretación de los resultados

\begin{tabular}{|c|c|c|c|}
\hline \multirow{2}{*}{ Áreas } & \multirow{2}{*}{$\begin{array}{l}\mathbf{N}^{o} \text { de } \\
\text { Ítems }\end{array}$} & \multicolumn{2}{|c|}{ Significado de los resultados } \\
\hline & & Baja puntuación & Alta puntuación \\
\hline Función Física & 10 & $\begin{array}{l}\text { Mucha limitación para realizar todas las } \\
\text { actividades físicas incluyendo bañarse o } \\
\text { vestirse debido a la salud }\end{array}$ & $\begin{array}{l}\text { Realiza todo tipo de actividades físicas, } \\
\text { incluyendo las más vigorosas, sin gran } \\
\text { limitación }\end{array}$ \\
\hline Rol Físico & 4 & $\begin{array}{l}\text { Problemas con el trabajo u otras } \\
\text { actividades diarias como resultado de su } \\
\text { salud física }\end{array}$ & $\begin{array}{l}\text { Ningún problema con el trabajo u otras } \\
\text { actividades diarias como resultado de la } \\
\text { salud física }\end{array}$ \\
\hline Dolor & 2 & Dolor muy severo y altamente limitante & $\begin{array}{l}\text { Ausencia de dolor o limitaciones debidas al } \\
\text { mismo }\end{array}$ \\
\hline Salud General & 5 & $\begin{array}{l}\text { El sujeto evalúa su salud como mala y cree } \\
\text { que probablemente empeorará }\end{array}$ & $\begin{array}{l}\text { Evalúa su salud personal como buena / } \\
\text { excelente }\end{array}$ \\
\hline Vitalidad & 4 & Cansancio y agotamiento todo el tiempo & $\begin{array}{l}\text { Lleno de entusiasmo y energía todo el } \\
\text { tiempo }\end{array}$ \\
\hline Función Social & 2 & $\begin{array}{l}\text { Interferencia frecuente y extrema con las } \\
\text { actividades normales debido a problemas } \\
\text { físicos y emocionales }\end{array}$ & $\begin{array}{l}\text { Realiza actividades sociales normales sin } \\
\text { interferencia debidas a problemas físicos o } \\
\text { emocionales }\end{array}$ \\
\hline Rol Emocional & 3 & $\begin{array}{l}\text { Problemas con el trabajo u otras } \\
\text { actividades diarias como resultado de } \\
\text { problemas emocionales }\end{array}$ & $\begin{array}{l}\text { Ningún problema con el trabajo u otras } \\
\text { actividades diarias como resultado de } \\
\text { problemas emocionales }\end{array}$ \\
\hline Salud Mental & 5 & $\begin{array}{l}\text { Sensación de nerviosismo y depresión todo } \\
\text { el tiempo }\end{array}$ & $\begin{array}{l}\text { Sensación de paz, felicidad y calma todo el } \\
\text { tiempo }\end{array}$ \\
\hline $\begin{array}{l}\text { Transición de } \\
\text { Salud }\end{array}$ & 1 & $\begin{array}{l}\text { Cree que su salud es mucho peor ahora que } \\
\text { hace un año }\end{array}$ & $\begin{array}{l}\text { Cree que su salud es mucho mejor ahora } \\
\text { que hace un año }\end{array}$ \\
\hline
\end{tabular}

\section{Análisis estadístico}

Para el análisis estadístico se utilizó el programa SPSS en su versión $10^{29}$. Para cada dimensión de salud se calcularon la media, la mediana, los percentiles, la desviación estándar y la proporción de los individuos con la puntuación máxima (efecto techo) y mínima (efecto suelo) para cada dimensión. Para permitir la comparabilidad con otras poblaciones de referencia los análisis se efectuaron sobre los sujetos de 18 ó más años de edad.

Se estudiaron las propiedades psicométricas de las escalas: la proporción de no respuestas, la fiabilidad a través del coeficiente alfa de Cronbach y la correlación de los ítems que componen cada dimensión/escala con la puntuación total de la misma.
Para la comparación de las medias de puntuaciones en función del sexo y el tiempo de permanencia en el PMM se utilizó el análisis de varianza, estimándose pruebas de Scheffe cuando la prueba $F$ resultaba significativa en casos de varibles con más de dos grupos. La asociación de las puntuaciones de las subescalas del SF-36 con la edad se realizó a través de análisis de correlación de Pearson. Asimismo, para la comparación de diferencias de medias encontradas en la muestra de estudio respecto a los valores poblacionales de referencia ${ }^{22,23}$ se utilizó la prueba t de Student, para lo cual se creó una base de datos en la hoja de cálculo Excel con los estadísticos oportunos (media, desviación estándar y número de casos) de cada una de las dimensiones del SF-36 y de cada una de las muestras de comparación, a los cuales se aplicaron los algoritmos propios de la prueba $\mathrm{t}^{30,31}$. 
Finalmente, se han tabulado para distintos periodos de permanencia en el PMM (Inicio, 1-6 meses, 7-12 meses, 13-18 meses, 19-24 meses y más de 25 meses) y para cada una de las dimensiones del SF-36, las puntuaciones correspondientes a los percentiles 5, 10, 25, $50,75,90$ y 95 .

\section{RESULTADOS}

La muestra de estudio $(n=726)$ está constituida en su mayoría por hombres $(80,2 \%)$ y presenta una edad media de 33,6 años [Rango: 18-59; Desviación Estándar (DE): 5,3]. La media de años desde que se iniciaron en el consumo de opiáceos es de 15,2 (DE: 5,2) años, con un rango intercuartil (RI) entre 11 y 19 años. La permanencia media en el programa de metadona es de 25,6 (DE: 23,6) meses (IR: 8-36); con una dosis media de 63,7 (DE: 29,2; IR: 40-80) mg/día. El 93,5\% de los sujetos han realizado algún tratamiento previo por su adicción a la heroína, siendo la modalidad más utilizada el dispositivo de salud ambulatorio (79,6\%), seguido de la unidad hospitalaria de desintoxicación $(38,7 \%)$ y de la comunidad terapéutica $(33,6 \%)$. Un $49,4 \%$ de los participantes presentan marcadores serológicos de VIH y un $83,3 \%$ de hepatitis (un 5,9\% VHC, un $14,9 \%$
VHB y un 62,5\% de ambos); el 15,9\% había sido diagnosticado en alguna ocasión de tuberculosis, el 26,3\% habían tenido sobredosis alguna vez y un $25,2 \%$ habían sido diagnosticados y tratados por otras patologías psiquiátricas.

En la tabla 1 se presentan las propiedades psicométricas de las diferentes escalas, asi como los paramétros descriptivos de cada escala del SF-36. Las tasas de no-respuesta a los ítems fueron consistentemente bajas e inferiores al $1 \%$. La fiabilidad alcanzada por todas las dimensiones fue superior a 0,70 , mostrando el valor mínimo en el caso de la dimensión de vitalidad $(=0,79)$ y su valor máximo en la escala de rol físico $(=0,92)$. La mayoría de las correlaciones de los elementos de una escala con el total de la misma mostraron rangos intermedios-altos; tan solo en el caso de la vitalidad el valor de correlación máximo es inferior a 0,70 .

La escala de función física obtuvo las puntuaciones más altas y la dimensión de salud general, seguidas de cerca por la vitalidad y la salud mental, las más bajas. Las escalas de transición del estado de salud y salud general mostraron valores de la mediana inferiores a 40. En todas las dimensiones se observaron puntuaciones máximas (100)

Tabla 1

Propiedades psicométricas de las dimensiones del SF-36 y valores descriptivos

\begin{tabular}{|l|c|c|c|c|c|c|c|c|c|}
\hline & $\begin{array}{c}\text { \% } \\
\text { No } \\
\text { respuesta }\end{array}$ & $\begin{array}{c}\text { Alfa de } \\
\text { Cronbach }\end{array}$ & $\begin{array}{c}\text { Correlación } \\
\text { del ítem con el } \\
\text { total. } \\
\text { Recorrido }\end{array}$ & $\bar{X}$ & D.E. & Md & $\begin{array}{c}\text { Rango } \\
\text { observado }\end{array}$ & $\begin{array}{c}\text { Efecto } \\
\text { Suelo }^{*}\end{array}$ & $\begin{array}{c}\text { Efecto } \\
\text { Techo }\end{array}$ \\
\hline Función Física & 0,1 & 0,89 & $0,48-0,80$ & 88,5 & 16,9 & 94,4 & $0-100$ & 0,3 & 38,1 \\
\hline Rol Físico & 0,0 & 0,92 & $0,78-0,85$ & 72,9 & 40,1 & 100 & $0-100$ & 19,4 & 63,5 \\
\hline Dolor & 0,0 & 0,84 & 0,75 & 75,1 & 28,8 & 88,9 & $0-100$ & 1,5 & 45,5 \\
\hline Salud General & 0,1 & 0,83 & $0,50-0,75$ & 43,9 & 26,6 & 40,0 & $0-100$ & 2,9 & 0,7 \\
\hline Vitalidad & 0,8 & 0,79 & $0,52-0,69$ & 54,6 & 22,7 & 55,0 & $0-100$ & 1,1 & 1,3 \\
\hline Función Social & 1,0 & 0,89 & 0,82 & 76,6 & 29,3 & 88,9 & $0-100$ & 3,3 & 47,7 \\
\hline Rol Emocional & 0,0 & 0,91 & $0,78-0,84$ & 71,4 & 41,5 & 100 & $0-100$ & 21,9 & 63,6 \\
\hline Salud Mental & 0,1 & 0,87 & $0,65-0,74$ & 55,9 & 23,2 & 60,0 & $0-100$ & 0,7 & 0,3 \\
\hline $\begin{array}{l}\text { Transición de } \\
\text { estado de salud }\end{array}$ & 0,4 & --- & --- & 63,9 & 75,0 & 26,2 & $0-100$ & 3,6 & 21,2 \\
\hline
\end{tabular}

$\bar{X}$ : Media; D.E.: Desviación Estándar; Md: Mediana; *: \% con la puntuación mínima (cero - 0); †: \% con la puntuación máxima (cien - 100) 
y mínimas (0), por lo que el rango observado se situó en 100. Los efectos suelo fueron inferiores al 4\%, salvo en el caso de las escalas de rol emocional $(21,9 \%)$ y rol físico $(19,4 \%)$. Los efectos techo fueron superiores al $60 \%$ en las escalas de rol físico y rol emocional, y cercanas o inferiores al $1 \%$ en las dimensiones de vitalidad, salud general y salud mental.

No se hallaron diferencias por sexo en las puntuaciones de las escalas del SF-36 y tampoco se encontraron asociaciones estadísticamente significativas entre la edad y las diferentes subescalas (correlaciones de Pearson entre 0,002 y 0,058), salvo en el caso de la función física $(\mathrm{r}=-0,097 ; \mathrm{p}=$ $0,009)$ y la salud mental $(\mathrm{r}=-0,110 ; \mathrm{p}=$ 0,003), en ambos casos de signo negativo.

El perfil de salud de los usuarios de PMMs respecto al ofrecido por las poblaciones de referencia se muestra en la tabla 2. El patrón observado en los tres grupos es similar, casi coincidente entre las dos poblaciones de referencia, si bien las puntuaciones de los usuarios de PMMs se muestran más bajas. Tanto atendiendo a la población general de la CAV como a la española, y a excepción del caso de la función física, en todas las dimensiones los usuarios de PMMs obtienen puntuaciones significativamente más bajas, indicativas de un peor estado de salud, alcanzando una magnitud considerable en las dimensiones de salud general, rol emocional y salud mental $(-23,4 ;-20,8$ y $-15,8$ puntos, respectivamente, cuando se compara con la población general de la CAV).

Las puntuaciones obtenidas por los usuarios de PMMs en función del tiempo de permanecia en los programas de metadona se exponen en la tabla 3. En todas las dimensiones se hallan diferencias estadísticamente significativas entre los grupos $(\mathrm{p}<0,001)$, y el resultado de las pruebas de Scheffe indican que los sujetos que inician el PMM muestran puntuaciones significativamente más bajas que aquellos otros que ya llevan algún tiempo en el programa. Las pruebas post-hoc (Scheffe) muestran diferencias entre grupos en las áreas de rol físico, salud mental y transición de la salud. En las dos

Tabla 2

Diferencias en las puntuaciones obtenidas en el SF-36 por la muestra de usuarios de PMM respecto a los valores de referencia de la población de la Comunidad Autónoma $\operatorname{Vasca}^{(22)}$ y de España ${ }^{(23)}$

\begin{tabular}{|c|c|c|c|c|c|c|c|c|}
\hline & \multicolumn{4}{|c|}{$\begin{array}{c}\text { Comparación con valores de referencia } \\
\text { CAV }\end{array}$} & \multicolumn{4}{|c|}{$\begin{array}{c}\text { Comparación con valores de referencia } \\
\text { España }\end{array}$} \\
\hline & $\bar{X}$ & Dif & $\mathbf{t}$ & $\mathbf{p}$ & $\bar{X}$ & Dif & $\mathbf{t}$ & $\mathbf{p}$ \\
\hline Función Física & 87,2 & 1,3 & 1,51 & n.s. & 84,7 & 3,8 & 4,18 & 0,001 \\
\hline Rol Físico & 87,3 & $-14,4$ & 9,66 & 0,001 & 83,2 & $-10,3$ & 7,51 & 0,001 \\
\hline Dolor & 79,6 & $-4,5$ & 3,87 & 0,001 & 79,0 & $-6,1$ & 5,35 & 0,001 \\
\hline Salud General & 67,6 & $-23,4$ & 23,90 & 0,001 & 58,3 & $-14,4$ & 16,48 & 0,001 \\
\hline \begin{tabular}{|l|} 
Vitalidad \\
\end{tabular} & 65,2 & $-10,6$ & 11,67 & 0,001 & 66,9 & $-12,3$ & 14,35 & 0,001 \\
\hline Función Social & 89,8 & $-13,2$ & 13,20 & 0,001 & 90,1 & $-13,5$ & 16,74 & 0,001 \\
\hline Rol Emocional & 92,2 & $-20,8$ & 15,40 & 0,001 & 86,6 & $-15,2$ & 12,68 & 0,001 \\
\hline \begin{tabular}{|l} 
Salud Mental \\
\end{tabular} & 71,7 & $-15,8$ & 18,14 & 0,001 & 73,3 & $-17,4$ & 22,17 & 0,001 \\
\hline
\end{tabular}

Dif.: Diferencia entre la media del grupo PMM y el grupo de referencia; t: Prueba t de Student para la comparación de medias entre grupos 
Tabla 3

Diferencias de medias de las puntuaciones del Cuestionario SF-36 en función del tiempo de permanencia en el Programa de Metadona

\begin{tabular}{|l|c|c|c|c|c|c|c|}
\hline & Inicio & $1-6$ meses & $7-12$ meses & $13-18$ meses & $19-24$ meses & $>25$ meses & $\begin{array}{c}\mathrm{F} \\
\mathrm{g}-1-5,714\end{array}$ \\
\hline Función Física & $69,7(26,2)^{\text {abcde }}$ & $89,4(15,1)^{\mathrm{a}}$ & $91,6(13,0)^{\mathrm{b}}$ & $91,5(14,1)^{\mathrm{c}}$ & $91,6(13,6)^{\mathrm{d}}$ & $90,1(13,7)^{\mathrm{e}}$ & $25,5^{*}$ \\
\hline Rol Físico & $33,5(42,1)^{\text {abcde }}$ & $87,6(29,7)^{\mathrm{af}}$ & $82,1(32,4)^{\mathrm{b}}$ & $82,1(33,0)^{\mathrm{c}}$ & $85,0(29,6)^{\mathrm{d}}$ & $69,6(42,1)^{\mathrm{ef}}$ & $24,7^{*}$ \\
\hline Dolor & $39,1(25,1)^{\text {abcde }}$ & $74,6(25,9)^{\mathrm{a}}$ & $78,7(23,7)^{\mathrm{b}}$ & $82,8(24,6)^{\mathrm{c}}$ & $85,3(24,9)^{\mathrm{d}}$ & $78,4(27,5)^{\mathrm{e}}$ & $36,0^{*}$ \\
\hline Salud General & $21,0(19,3)^{\text {abcde }}$ & $43,2(28,2)^{\mathrm{a}}$ & $47,7(25,7)^{\mathrm{b}}$ & $48,6(26,5)^{\mathrm{c}}$ & $53,5(24,6)^{\mathrm{d}}$ & $44,5(25,6)^{\mathrm{e}}$ & $16,1^{*}$ \\
\hline Vitalidad & $29,8(17,9)^{\text {abcde }}$ & $54,2(21,8)^{\mathrm{a}}$ & $57,9(19,1)^{\mathrm{b}}$ & $61,3(21,5)^{\mathrm{c}}$ & $58,2(22,8)^{\mathrm{d}}$ & $57,1(21,6)^{\mathrm{e}}$ & $25,6^{*}$ \\
\hline Función Social & $52,0(38,0)^{\text {abcde }}$ & $77,3(28,4)^{\mathrm{a}}$ & $84,7(22,5)^{\mathrm{b}}$ & $81,2(25,6)^{\mathrm{c}}$ & $81,7(26,2)^{\mathrm{d}}$ & $76,6(27,7)^{\mathrm{e}}$ & $15,2^{*}$ \\
\hline Rol Emocional & $34,1(43,0)^{\text {abcde }}$ & $82,6(36,0)^{\mathrm{a}}$ & $82,8(32,3)^{\mathrm{b}}$ & $74,5(39,7)^{\mathrm{c}}$ & $76,6(39,0)^{\mathrm{d}}$ & $71,5(41,5)^{\mathrm{e}}$ & $17,8^{*}$ \\
\hline Salud Mental & $30,0(18,0)^{\text {abcde }}$ & $51,1(23,9)^{\mathrm{af}}$ & $55,9(23,4)^{\mathrm{b}}$ & $59,7(20,9)^{\mathrm{c}}$ & $61,6(19,4)^{\mathrm{d}}$ & $61,4(20,8)^{\mathrm{ef}}$ & $29,6^{*}$ \\
\hline $\begin{array}{l}\text { Transición de } \\
\text { estado de salud }\end{array}$ & $55,3(27,3)^{\mathrm{ab}}$ & $57,3(28,2)^{\mathrm{c}}$ & $68,1(25,9)^{\mathrm{a}}$ & $73,2(24,5)^{\mathrm{bcd}}$ & $66,1(23,7)$ & $62,3(25,6)^{\mathrm{d}}$ & $6,3^{*}$ \\
\hline
\end{tabular}

abad: Prueba de Scheffe - Letras iguales indican la existencia de diferencias estadísticamente significativas entre las medias señaladas. F:Prueba $F$ de Scenecor (Análisis de Varianza) / g.l.: Grados de Libertad $*: \mathrm{p}<0,001$

primeras dimensiones se observa una menor puntuación en los sujetos con más de 25 meses en el programa respecto a los que llevan entre 1 y 6 meses. En el caso de la escala de transición de salud los sujetos que inician el PMM presentan puntuaciones más bajas que los que llevan entre 7-12 y 13-18 meses; los sujetos con 1-6 meses de permanencia ofrecen puntuaciones más bajas que los que llevan 13-18 meses, y este grupo presenta puntuaciones más altas que las observadas entre los usuarios con permanencia superior a los 25 meses.

En la tabla 4 se presentan diferentes percentiles obtenidos para cada dimensión de salud en función del tiempo de permanencia en el PMM. Estos son los valores de referencia a utilizar para evaluar las puntuaciones obtenidas en la práctica clínica entre usuarios de PMM.

\section{DISCUSIÓN}

Los datos presentados correponden a los resultados de la aplicación de la versión en castellano del cuestionario de salud SF-36 a una amplia muestra representativa de la población de personas con adicción a opiá- ceos en tratamiento en un programa de mantenimiento con metadona de la Comunidad Autónoma Vasca. La aplicación del instrumento de medida y sus características psicométricas se han mostrado adecuadas y muy similares a las obtenidas en el estudio de adaptación a población española ${ }^{22,23}$. La proporción de información perdida no supera el $1 \%$, con un tiempo medio de cumplimentación de 9,3 minutos. Respecto a la fiabilidad de las dimensiones que agrupa el SF-36 se han obtenido unos índices de consitencia interna (alpha de cronbach) por encima de 0,70-valor mínimo recomendado para realizar comparaciones de grupos $^{32}-$, e incluso superiores al valor de 0,80 . En concreto, tres dimensiones alcanzan valores próximos a 0,90 y dos superan dicho valor, el cual se ha sugerido como el nivel de precisión necesario para poder utilizar las puntuaciones del cuestionario a nivel individualizado ${ }^{13,33,34}$. Estudios previos han mostrado la equivalencia cultural y adaptacón al castellano del SF36 , tanto en población general ${ }^{22,23}$ como en muestras de pacientes ${ }^{25}$, y los resultados obtenidos en el presente estudio y otros afines ${ }^{15,35,41}$ parecen sugerir que este cuestionario también es aplicable para la valoración de personas con problemas de adicción. 
Tabla 4

Percentiles de las puntuaciones del Cuestionario SF-36 en función del tiempo de permanencia en el Programa de metadona

\begin{tabular}{|c|c|c|c|c|c|c|c|}
\hline & & Inicio & 1-6 meses & 7-12 meses & 13-18 meses & 19-24 meses & $>25$ meses \\
\hline \multirow{7}{*}{$\begin{array}{r}\text { Función } \\
\text { Física }\end{array}$} & Percentil 5 & 22,2 & 50,0 & 61,4 & 66,7 & 58,6 & 65,5 \\
\hline & Percentil 10 & 27,7 & 72,2 & 77,8 & 73,3 & 83,3 & 77,8 \\
\hline & Percentil 25 & 50,0 & 83,3 & 88,9 & 88,9 & 88,9 & 88,9 \\
\hline & Percentil 50 & 72,2 & 94,4 & 94,4 & 94,4 & 94,4 & 94,4 \\
\hline & Percentil 75 & 94,4 & 100 & 100 & 100 & 100 & 100 \\
\hline & Percentil 90 & 100 & 100 & 100 & 100 & 100 & 100 \\
\hline & Percentil 95 & 100 & 100 & 100 & 100 & 100 & 100 \\
\hline \multirow[t]{7}{*}{ Rol Físico } & Percentil 5 & 0,0 & 0,0 & 0,0 & 0,0 & 0,0 & 0,0 \\
\hline & Percentil 10 & 0,0 & 40,0 & 25,0 & 5,0 & 27,5 & 0,0 \\
\hline & Percentil 25 & 0,0 & 100 & 75,0 & 75,0 & 75,0 & 25,0 \\
\hline & Percentil 50 & 0,0 & 100 & 100 & 100 & 100 & 100 \\
\hline & Percentil 75 & 50,0 & 100 & 100 & 100 & 100 & 100 \\
\hline & Percentil 90 & 100 & 100 & 100 & 100 & 100 & 100 \\
\hline & Percentil 95 & 100 & 100 & 100 & 100 & 100 & 100 \\
\hline \multirow[t]{7}{*}{ Dolor } & Percentil 5 & 11,1 & 31,1 & 33,3 & 33,3 & 22,2 & 22,2 \\
\hline & Percentil 10 & 11,1 & 44,4 & 44,4 & 44,4 & 34,4 & 33,3 \\
\hline & Percentil 25 & 22,2 & 55,5 & 66,7 & 66,7 & 77,8 & 55,5 \\
\hline & Percentil 50 & 33,3 & 77,8 & 88,9 & 100 & 100 & 100 \\
\hline & Percentil 75 & 55,5 & 100 & 100 & 100 & 100 & 100 \\
\hline & Percentil 90 & 77,8 & 100 & 100 & 100 & 100 & 100 \\
\hline & Percentil 95 & 88,9 & 100 & 100 & 100 & 100 & 100 \\
\hline \multirow{7}{*}{$\begin{array}{r}\text { Salud } \\
\text { General }\end{array}$} & Percentil 5 & 0,0 & 0,0 & 10,0 & 10,5 & 10,0 & 5,0 \\
\hline & Percentil 10 & 0,0 & 5,0 & 15,0 & 15,0 & 16,0 & 15,0 \\
\hline & Percentil 25 & 5,0 & 20,0 & 25,0 & 25,0 & 35,0 & 25,0 \\
\hline & Percentil 50 & 15,0 & 40,0 & 45,0 & 45,0 & 50,0 & 40,0 \\
\hline & Percentil 75 & 30,0 & 65,0 & 68,7 & 67,8 & 70,0 & 65,0 \\
\hline & Percentil 90 & 50,0 & 85,0 & 85,0 & 90,0 & 89,5 & 85,0 \\
\hline & Percentil 95 & 65,0 & 91,0 & 90,0 & 95,0 & 90,0 & 90,0 \\
\hline \multirow[t]{7}{*}{ Vitalidad } & Percentil 5 & 0,0 & 18,0 & 20,0 & 25,2 & 10,0 & 20,0 \\
\hline & Percentil 10 & 10,0 & 25,0 & 30,0 & 30,5 & 21,0 & 30,0 \\
\hline & Percentil 25 & 15,0 & 35,0 & 45,0 & 45,0 & 48,7 & 40,0 \\
\hline & Percentil 50 & 25,0 & 55,0 & 60,0 & 60,0 & 65,0 & 55,0 \\
\hline & Percentil 75 & 40,0 & 70,0 & 70,0 & 80,0 & 71,2 & 75,0 \\
\hline & Percentil 90 & 50,0 & 80,0 & 80,0 & 90,0 & 85,0 & 85,0 \\
\hline & Percentil 95 & 65,0 & 86,0 & 85,0 & 94,7 & 95,0 & 90,0 \\
\hline \multirow{7}{*}{$\begin{array}{r}\text { Función } \\
\text { Social }\end{array}$} & Percentil 5 & 0,0 & 22,2 & 33,3 & 23,3 & 22,2 & 18,9 \\
\hline & Percentil 10 & 0,0 & 27,8 & 44,4 & 35,5 & 33,3 & 33,3 \\
\hline & Percentil 25 & 22,2 & 55,5 & 69,4 & 66,7 & 66,7 & 55,5 \\
\hline & Percentil 50 & 44,4 & 94,4 & 100 & 100 & 100 & 88,9 \\
\hline & Percentil 75 & 100 & 100 & 100 & 100 & 100 & 100 \\
\hline & Percentil 90 & 100 & 100 & 100 & 100 & 100 & 100 \\
\hline & Percentil 95 & 100 & 100 & 100 & 100 & 100 & 100 \\
\hline \multirow{7}{*}{$\begin{array}{r}\text { Rol } \\
\text { Emocional }\end{array}$} & Percentil 5 & 0,0 & 0,0 & 0,0 & 0,0 & 0,0 & 0,0 \\
\hline & Percentil 10 & 0,0 & 0,0 & 3,3 & 0,0 & 0,0 & 0,0 \\
\hline & Percentil 25 & 0,0 & 100 & 66,6 & 66,7 & 66,7 & 33,3 \\
\hline & Percentil 50 & 0,0 & 100 & 100 & 100 & 100 & 100 \\
\hline & Percentil 75 & 66,7 & 100 & 100 & 100 & 100 & 100 \\
\hline & Percentil 90 & 100 & 100 & 100 & 100 & 100 & 100 \\
\hline & Percentil 95 & 100 & 100 & 100 & 100 & 100 & 100 \\
\hline \multirow{7}{*}{$\begin{array}{r}\text { Salud } \\
\text { Mental }\end{array}$} & Percentil 5 & 4,0 & 11,2 & 16,0 & 20,4 & 16,0 & 20,0 \\
\hline & Percentil 10 & 8,0 & 18,4 & 24,0 & 28,8 & 32,8 & 36,0 \\
\hline & Percentil 25 & 20,0 & 36,0 & 40,0 & 46,0 & 51,0 & 48,0 \\
\hline & Percentil 50 & 28,0 & 48,0 & 60,0 & 60,0 & 64,0 & 64,0 \\
\hline & Percentil 75 & 40,0 & 72,0 & 72,0 & 76,0 & 76,0 & 76,0 \\
\hline & Percentil 90 & 56,0 & 84,0 & 84,0 & 84,0 & 84,0 & 88,0 \\
\hline & Percentil 95 & 68,0 & 88,0 & 92,0 & 92,0 & 88,0 & 92,0 \\
\hline
\end{tabular}


Los resultados obtenidos en las diferentes dimensiones del SF-36, así como su comparación con datos normativos poblacionales, nos llevan a concluir que el estado de salud de las personas en PMM está seriamente comprometido. Respecto a su grupo normativo de referencia, el de la Comunidad Autónoma Vasca $^{23}$, salvo en la función física, se han hallado diferencias estadísticamente significativas indicativas de un peor estado de salud en todas las dimensiones, alcanzando una diferencia de más de 10 puntos en seis de ellas. El caso más extremo se halla en la apreciación del estado general de salud, el cual es valorado por los usuarios de PMM 23,2 puntos por debajo del valor mostrado por el grupo normativo. De forma contraria a lo que cabría esperar no se han hallado diferencias por sexo, como ocurre en poblacion general $^{22,23}$, por lo que la valoración de salud que hacen hombres y mujeres con trastornos de dependencia a opiáceos presenta similares características. Respecto a la edad se ha observado una asociación negativa con la funcion física y la salud mental, de forma que la mayor disfunción en estas áreas se observaría entre las personas de más edad y, por ende, entre las que llevan un mayor número de años con el problema de adicción.

Cinco de las ocho dimensiones muestran puntuaciones medias globales por encima de 70 , mientras las tres restantes ofrecen valores entre 43,9 y 55,9, indicando un mayor compromiso de la salud. En concreto, las personas en tratamiento en un PMM expresan una valoración positiva, o mejor dicho más adecuada, de su función física que de su estado de salud general, vitalidad o salud mental. Estas tres dimensiones serían las que, en promedio, los usuarios de PMM perciben como más deficitarias. No obstante, si atendemos a los efectos suelo, son las limitaciones del desempeño del rol por causa de problemas físicos y/o emocionales las que producen un mayor número de casos con limitación extrema ya que, respectivamente, un $19,4 \%$ y un $21,9 \%$ de los casos han ofre- cido puntuaciones de cero, indicativas del mayor grado de limitación posible.

Estos resultados son similares a los hallazgos encontrados en otros estudios sobre poblaciones análogas que han utilizado el SF-36 $6^{36,42}$. En todos los casos se observan en las personas en tratamiento por adicción a opiáceos índices de percepción de salud por debajo de sus poblaciones de referencia y con valoraciones de la calidad de vida similares a las ofrecidas por otros grupos con enfermedades crónicas (somáticas y/o psiquiátricas $)^{25,35,39}$. De igual modo, en todos los estudios las áreas del SF-36 que muestran mayor déficit son las de vitalidad, salud mental y percepción general de la salud, las cuales se asocian a una mayor prevalencia de enfermedades infecciosas (VIH y VHC) y comorbilidad psiquiátrica ${ }^{35,42}$.

Las investigaciones sobre calidad de vida en usuarios de PMM $^{35,43}$ han reflejado una mejora notable de la misma tras su ingreso y mantenimiento en este tipo de programas. Nuestros datos, basados en un diseño transversal, se muestran limitados para ofrecer valoraciones de cambio consecuentes a la permanencia en el PMM, pero sí permiten observar diferencias en la percepción del estado de salud entre los que inician el programa y aquellos otros usuarios que ya llevan un tiempo en tratamiento, sugerentes de un aumento de la calidad de vida. La mayor diferencia se aprecia precisamente entre el grupo de sujetos que inicia el tratamiento y los que llevan seis meses; a partir de ese momento las puntuaciones medias de la mayoría de las áreas del SF-36 tienden a mostrarse equivalentes. La hipótesis subyacente es que el PMM propicia la mejoría y estabilización del estado de salud y áreas conexas, y si bien esta hipótesis no puede obtener respuesta con los datos de nuestro estudio, sí ha sido contrastada a través de otros estudios realizados dentro y fuera de nuestro contexto geográfico ${ }^{39,40}$.

A la espera de mayor evidencia que reafirme la validez de este efecto de cambio en la 
mejoría de la calidad de vida percibida en PMM, los datos de nuestro estudio se proponen como báremo normativo con el que comparar los resultados esperados para un caso dado cuando se sitúe en un determinado tiempo de permanencia en un PMM. La obtención de valores poblacionales de referencia permite establecer criterios para la interpretación de las puntuaciones a partir de las que se utilizan como normativas ${ }^{44}$. Las puntuaciones del SF-36 no tienen significado por sí mismas. Como la mayoría de las medidas de salud percibida, deben ser interpretadas como mayor o menor presencia de la característica evaluada, no debiendo ser interpretadas de forma aislada, sino en comparación con la distribución de las puntuaciones de un grupo de referencia ${ }^{44}$. El análisis de la distribución de estas puntuaciones en una muestra representativa amplia permite comparar subgrupos de población en función de una determinada característica; el sexo y/o la edad de forma genérica, o la permanencia en el PMM en nuestro caso. Además, facilita la evaluación de la relación entre las diferencias estadísticas y la magnitud o el tamaño del efecto ${ }^{45}$ a partir de su relación con variaciones en el estado de salud, o antes y después de intervenciones sanitarias $^{46,47}$

En este estudio se ha administrado la versión española del SF-36 a una muestra representativa de usuarios de PMM del País Vasco. Los valores de las medias y percentiles poblacionales presentados para cada dimensión del SF-36 podrán tomarse como referencia para comparar con los datos obtenidos en futuros estudios donde se administre el instrumento a este tipo de población. Si se toma como ejemplo la distribución de las puntuaciones en la dimensión de salud mental del SF-36 a los 12 meses (tabla 4), se observa que el 50\% de los usuarios de PMM presentan puntuaciones inferiores a 60 . Por el contrario, un $25 \%$ se sitúa por debajo de 40 (percentil 25) o por encima de 72 (percentil 75). Asimismo, un usuario de PMM con una puntuación de 84 en salud mental se sitúa en el percentil 90, e indica que tan sólo un $10 \%$ de sujetos del grupo tendría puntuaciones por encima de la suya. Esta puntuación sería deseable para la mayoría de las personas en tratamiento a los 12 meses y podría plantearse como meta del tratamiento en ese periodo. Por otro lado, la puntuación obtenida por un sujeto puede ser valorada en función de la magnitud de las diferencias, definida como la «distancia» o diferencia estandarizada de las puntuaciones ${ }^{45}$. Siguiendo con el ejemplo propuesto, la diferencia de puntuación de un sujeto que obtiene una puntuación de 35 en la dimensión de salud mental a los 12 meses respecto a la media del grupo ( $M=55,9$; tabla 3$)$ equivaldría a un tamaño del efecto de 0,89 (35 - 55,9 $/ 23,4)$, que ha de interpretarse como elevado. Planteado desde otro punto de vista, una diferencia de 18,7 puntos representaría un tamaño de efecto de 0,8 que se sugiere como elevada, una puntuación de 11,7 representa una diferencia moderada $(0,5)$ y la diferencia mínima esperada con relevancia clínica (tamaño del efecto de 0,2$)^{48}$ sería de un 4,7 puntos diferenciales. Para un más extenso conocimiento del uso e interpretación de las normas poblacionales pueden consultarse diferentes manuales al uso ${ }^{16,24,33,44,46,49} \mathrm{o}$ bien acudir a las especificaciones ofrecidas por el grupo de Alonso ${ }^{22}$ respecto a las normas para población general del SF-36.

En el ámbito de los trastornos adictivos la introducción de la calidad de vida como indicador de resultados es relativamente reciente, y en nuestro país la producción científica en esta materia es todavía escasa y se ha centrado en un pequeño grupo de equi$\operatorname{pos}^{15,50}$. No obstante, sí se ha resaltado la conveniencia y oportunidad de considerar este concepto como variable de resultado a incorporar en los estudios de evaluación terapéutica en toxicomanías ${ }^{5,6,50,51}$. Los cambios en la calidad de vida percibida por los pacientes pueden servir de medida de los resultados, tanto en la práctica clínica como en la investigación, utilizándose, por ejemplo, metodologías cuasiexperimentales 
basadas en la existencia de datos de referencia establecidos a partir de muestras $\operatorname{amplias}^{52}$. Asimismo, la valoración de la calidad de vida puede constituir un parámetro clave en los estudios de coste-beneficio y contribuir así a la optimización de los recursos en el ámbito de las drogodependencias, como ya se ha hecho respecto a otras patologías $^{53}$.

En resumen, los resultados del presente estudio confirman la fiabilidad y la validez de la versión española del SF-36 en una muestra representativa de usuarios de Programas de Mantenimiento con Metadona del País Vasco, y sugieren que el instrumento puede ser utilizado en estudios clínicos. Asimismo, facilitarán la interpretación de las puntuaciones del SF-36 en personas con dependencia a opiáceos y serán de utilidad para futuros estudios de valoración de este tipo de programas. No obstante, es necesario proseguir el análisis de los factores que intervienen en el estado de salud durante la permanencia en programas de tratamiento con metadona, analizar el comportamiento de los perfiles de salud en la población de personas con adicción a drogas, y evaluar la sensibilidad a los cambios de este instrumento de medida en este tipo de poblaciones.

\section{BIBLIOGRAFÍA}

1. Marsch LA. The effcacy of methadone maintenance interventions in reducing illicit opiate use, HIV risk behavior and criminality: a meta-analysis. Addiction 1998; 93: 515-32.

2. Ward J, Hall W, Mattick RP. Role of maintenance treatment in opioid dependence. Lancet 1999; 353: 221-6.

3. Van Beusekom I, Iguchi M. A review of recent advances in knowledge about methadone maintenance treatment. RAND Corporation; 2001. [citado 14 de abril de 2004]. Disponible en: http://www. rand.org/publications/ MR/MR1096.

4. Naciones Unidas. Abuso de drogas: Tratamiento y rehabilitación. Guía práctica de planificación y aplicación. Nueva York: Oficina contra la Droga y el Delito; 2003.

5. Fernández-Miranda JJ. Efectividad de los programas de mantenimiento con metadona. Una revisión de los resultados de los estudios de evaluación. Med Clin (Barc) 2001; 166: 150-4.

6. Iraurgi I. Calidad de vida en programas de metadona. Pub Of SEISIDA 2000; 11(4): 237-9.

7. Patrick DL, Peach H, Gregg I. Disablement and care: a comparison of patient views and general practitioner knowledge. J R College Gen Pract 1982; 32: 429-34.

8. Larson CO, Nelson EC, Gustafson D, Batalden PB. The relationship between meeting patients' information needs and their satisfaction with hospital care and general health status outcomes. Int J Qual Health Care 1996;8:447-56.

9. Patrick D, Deyo R. Generic and diseasespecific measures in assesing health status and quality of life. Med Care 1989; 27: S217-S32.

10. Guyatt GH. A taxonomy of health status instruments. J Rheumatol 1995; 22(6): 1188-90.

11. Bowling A. Measuring disease. Buckingham-Philadelphia: Open University Press; 1995.

12. Badia X, Carné X. La evaluación de la calidad de vida en el contexto del ensayo clínico. Med Clín (Barc) 1998; 110: 550-6.

13. Alonso J. Medidas de salud. En: Del Llano J, et al (Eds.). Gestión sanitaria: innovaciones y desafíos. Barcelona: Masson; 1997.

14. Mira JJ, Rodríguez Marín J, Tirado S, Sitges E. Semejanzas y diferencias entre la satisfacción y calidad percibida. Rev Calidad Asistencial 2000; 15: $36-42$.

15. Iraurgi I. Instrumentos de Evaluación de la Calidad de Vida Relacionada con la Salud en Toxicomanías. En: Iraurgi I, González-Saiz F. (Eds.). Instrumentos de evaluación en drogodependencias. Madrid: Aula Médica; 2002: 481-511.

16. Ware JE, Snow KK, Kosinski M, Gandek B. SF-36 Health Survey. Manual and Interpretation Guide. Boston: The Health Institute, New England Medical Center; 1993.

17. Ware JE, Sherbourne CD. The MOS 36-item short form health survey (SF-36). I. Conceptual framework and item selection. Med Care 1992; 30: 47383. 
18. Mchorney CA, Ware JE, Raczek AE. The MOS 36Item Short-Form Health Survey (SF-36). II. Psycometric and Clinical test of validity in measuring physical and mental health construct. Med Care 1993; 3: 247-63.

19. Mchorney Ca, Ware Je, Lu Jfr, Sherbourne CD. The MOS 36-item short-form Health Survey (SF-36). III. Test of data quality, scaling assumptions, and realibility accross diverse patient groups. Med Care 1994; 32: 40-66.

20. Brazier JE, Harper R, Jones NMB, O'Cathain A, Thomas KJ, Usherwood T, Westlake L. Validating the SF-36 Health Survey questionnaire: new outcome measure for primary care. BMJ 1992; 305: 160-

21. Alonso J, Prieto L, Antó JM. La versión española del SF-36 Health Survey (Cuestionario de salud SF-36): un instrumento para la medida de los resultados clínicos. Med Clin (Barc) 1995; 104: 771-6.

22. Alonso J, Regidor E, Barrio G, Prieto L, Rodríguez C, De La Fuente L. Valores poblacionales de referencia de la versión española del Cuestionario de salud SF-36. Med Clin (Barc) 1998; 111: 410-6.

23. Anaitua C, Quintana JM. Valores poblacionales del índice de salud SF-36 en el País Vasco: importancia y aplicación en la práctica clínica. Osasunkaria 1999; 17: 10-7.

24. Gandek B, Ware JE Jr. (Eds). Translating funtional health and well-being: International Quality of Life Assessment (IQOLA) Project studies of the SF-36 Health Survey. Special Issue. J Clin Epidemiol 1998; 51: 891-1214.

25. Ruiz de Velasco I, Quintana JM, Padierna JA, Aróstegui A , Bernal A, Pérez-Izquierdo J , Ojanguren JM, Anitua C , González N, Etxeberria Y. Validez del cuestionario de calidad de vida SF-36 como indicador de resultados de procedimientos médicos y quirúrgicos. Rev Calidad Asistencial 2002;17(4):206-12.

26. Osakidetza - Servicio Vasco de Salud. Memoria de Actividad. Salud Mental y Asistencia Psiquiátrica Extrahospitalaria. Informe Interno; 1999.

27. Badia X, Salamero M, Alonso J, Ollé A. La medida de la salud. Guía de escalas de medición en español. $2^{a}$ edición. Barcelona: Edimac; 1999.

28. IMIN - Institut Municipal d'Investigació Mèdica. Manual de puntuación de la versión española del Cuestionario de Salud SF-36. [Citado 14 de abril de 2004]. Disponible en: http://www.imim.es/ $\mathrm{imim} / \mathrm{cas} / \mathrm{c}-\mathrm{QQV} . h \mathrm{tm}$
29. Norusis MJ / SPSS Inc. Statistical Package for the Social Sciences. Release 10. Chicago: SPSS Inc; 2000 .

30. Black TR. Doing quantitative research in the social sciences. An integrated approach to reserch design, measurement and statistics. London: Sage; 1999.

31. Parra I. Estadística empresarial con Microsoft Excel. Problemas de inferencia estadística. 2da edición. Madrid : Editorial AC; 2003.

32. Campbell DT, Fiske DW. Convergent and discriminant validation by the multitrait-multimethods matrix. Psychol Bull 1959; 56: 81-105.

33. Nunnally JC, Bernstein IL. Teoría psicométrica. México: McGraw Hill; 1995.

34. McHorney CA, Tarlov AR. Individual-patient monitoring in clinical practice: are avalaible health status surveys adequate?. Qual Life Res 1995; 4: 293-307.

35. Ryan CF, White JM. Health status at entry to methadone maintenance treatment using the SF-36 health survey questionnaire. Addiction 1996; 91 (1): 39-45.

36. Fernández-Miranda JJ, González MP, Saiz PA, Bobes J. Calidad de vida y severidad de la adicción en heroinómanos en mantenimiento prolongado con metadona. Adicciones 1999; 11: 43-52.

37. Iraurgi I, Casas A, Celorio MJ, Díaz-Sanz M. Calidad de vida relacionada con la salud en usuarios de un programa de metadona. Adicciones 1999; 24 . 131-47.

38. Okano GJ, Malone DC, Billups SJ, Carter BL, Sintek CD, Covey D, Mason B, Jue S, Carmichael J, Guthrie K, Dombrowski R, Geraets DR, Amato MG. Reduced Quality of Life in Veterans at Risk for Drug-Related Problems. Pharmacotherapy 2001; 21(9):1123-9.

39. Freeman K. New South Wales Drug Court Evaluation: Interim Report On Health And Well-Being Of Participants. Crime and Justice Bulletin, 2001; 53, New South Wales Bureau of Crime Statistics and Research, Sydney. [citado 14 de abril de 2004]. Disponible en: http://www.lawlink.nsw.gov.au/ bocsar1.nsf/files/ cjb53.pdf/\$file/cjb53.pdf.

40. Iraurgi I, Casas A, Celorio MJ, García-Serrano G, Hormaza M. Estado de salud y calidad de vida en usuarios de un programa de mantenimiento con metadona. III Congreso Virtual de Psiquiatría, 2002. [citado 14 de abril de 2004]. Disponible en: hppt://www.psiquiatria.com/interpsiquis 2002/5590.

Rev Esp Salud Pública 2004, Vol. 78, N. ${ }^{\circ} 5$ 
41. Puigdollers E, Cots F, Brugal MT, Torralba L, Domingo-Salvany A. Programas de mantenimiento de metadona con servicios auxiliares: un estudio de coste-efectividad. Gac Sanit 2003; 17(2): 123 30 .

42. Markez I, Iraurgi I, Poó M, Ordieres E, Del Arco J, Presser E, et al. Los programas de manetenimiento con metadona en la Comunidad Autónoma del País Vasco. Bilbao: Consejo de Colegios Farmaceúticos de Euskadi; 2000.

43. Torrens M, San L, Martínez A, Domingo-Salvany A, Alonso J Use of the Nottingham Health Profile for measuring health status of patients with methadone maintenance treatment. Addiction 1997; 92: 707-16.

44. Gandek B, Ware JE. Methods for validating and norming translations of health status questionnaires: the IQOLA project approach. J Clin Epidemiol 1998; 51: 953-9.

45. Coe R. What is an 'effect size'?: a guide for users. [citado 14 de abril de 2004]. Disponible en: http://cem.dur.ac.uk/ ebeuk/research/effectsize/ Esguide.htm.

46. Scientific Advisory Commitee of the Medical Outcomes Trust. Assessing health status and quality of life instruments: attributes and review criteria. Qual Life Res 2002; 11: 193-205.
47. Higginson IJ, Carr AJ. Using quality of life measures in clinical setting. BMJ 2001; 322: 1297-300.

48. Cohen J. Statistical power analysis for behavioral sciences. Hillsdale: Lawrence Erlbaum Associates; 1988.

49. American Psychological Association. Standards for educational and psychological testing. Washington, DC: American Psychological Association, 1985.

50. Fernández-Miranda JJ. La calidad de vida en adicciones: una medida de la efectividad de los tratamientos. Anales de Psiquiatría 2003; 19, 9: 377-84.

51. Iraurgi I. La calidad de vida como indicador de resultados en la clínica de las drogodependencias. Lasdrogas.info. Instituto para el Estudio de las Adicciones, 1999. [citado 14 Junio 2004]. Disponible en: http://www.ieanet.com/boletin/ opinion.html?o=25.

52. Iraurgi I. Cuestiones metodológicas en la evaluación de programas terapéuticos. Trastornos Adictivos 2000; 2, 2: 99-113.

53. Badia X. La medida de la calidad de vida relacionada con la salud en la IRS. En: Avances en Investigación de Resultados en Salud, 119-127. Barcelona: Ecdimac / Novartis. 